\title{
The several combining "body" fault interpreting technique that is used in bayanchagan region
}

\author{
Haifeng Chen, Xixin Wang, Zhendong Lei \\ 1 Northeast Petroleum University The formation mechanism of oil and gas reservoir and resource evaluation \\ Heilongjiang Province key laboratory, Daqing, Heilongjiang, China, 163318
}

\begin{abstract}
Fault is one of most common tectonic deformation style in a sedimentary basin,the relationship between the fault and Oil-gas reservoirs is research hot spot of Petroleum Geology all the time. Fault may control the formation and development of sedimentary basin, restrict the filling process of deposition, thus affect the geological factors and geological processes of oil and gas reservoirs. So before exploration and development to the study area, the study to the fault and the fracture system is the most basic work, and the fault interpretation is particularly important.The several combining "body" faults'interpreting technique (eigenvalue of coherency cub technique、variance of coherency cub technique、 azimuth of coherency cub technique、 dip testing technique) is used in this region. This test introduces the characteristics and the extracting methods of the several coherency, In the tectonic interpretation process of this region, we explicated complex faults and the fracture feature description more convenient.
\end{abstract}

Keywords: the bayanchagan region fault interpretion several body combine

\section{INTRODUCTION}

The central sag in Songliao basin area is the main exploration area[1,2], where there are several level two tectonic units, and the Longhupao-Daan terrace is one of them, which is located in the excessive part between the central depression area and the western slope zone. The bayachagan region is located in the Longhupao-Daan terrace and Gulong depression, stretch across the two tectonic units, the Qijia- Gulong depression and the Longhupao-Daan terrace. Gaotaizi and Putaohua Oilfield of Daqing placanticline in the East,and the west is Talaha Oilfield, and the south is Puxi Oilfield, and the north is Longnan Oilfield. The bayachagan region' structural trend is SN-NNE, and faults, local structure is developed. In order to understand the fanlt system characteristics influence on the the remaining oil distribution further, we must interpretat the faults finely.

\section{FINE INTERPRETATION OF FAULTS}

The fault is the the most common tectonic deformation style in sedimentary basin ${ }^{[3,4]}$, the relationship between faults and oil and gas reservoirs is research hot spot of Petroleum Geology all the time. Fault may control the formation and development of sedimentary basin, restrict the filling process of deposition, thus affect the geological factors and geological processes of oil and gas reservoirs. Faults can form traps,also can form traps together with fold, monoclinic and uplift and so om. In fact, most structural trap oil-gas reservoirs are reformed by faults. Fault is the pathway of hydrocarbon migration, whether the activity of faults is the key oil and gas reservoir adjustment and preservation again. Fault system, fault zone, especially deep fracture zone control the distribution of oil and gas fields in the basin, the fault of different scale may control the type and scale of oil and gas fields, so in the regional exploration process, the detailed study of fault system is particularly important.

Faults is often manifested by the phenomenon in the seismic time section: the reflection wave phase axis dislocation, the reflection wave phase axis suddenly increase or decrease or disappear, a sudden change in wave group interval, the reflection events shape mutation, reflex disorder or blank, standard of reflection events, twisted, bifurcation, with strong phase transformation, the presence of abnormal wave and so on ${ }^{[5]}$.

This study used the multi-body combined, that is the eigenvalue related body, variance, azimuth coherence, dip angle detection, to interpret faults.

The eigenvalue of coherent technology is a method for computing the quantitative waveform similarity, which is achieved by the definition of "global space-time and calculated of angle and azimuth angle ${ }^{[6]}$. Through this kind of method, the fault and fracture system may image in space or plane, without interpreting faults subjectively in the chosen section and combing faults in plane ${ }^{[7]}$.

Variance cube technology is a new seismic data interpretation technology developed in recent years, that the technology and the coherence technique are used in conjunction can improve the accuracy of fault interpretation further ${ }^{[8]}$. Analysis of variance of probability is the concept source of the variance technique, the 
calculation of variance is through the calculating the average of each sampling point within the time window of seismic trace and sampling point in all the time window, thus we can get abnormal seismic reflection caused by discontinuities or abnormal of geological properties. It can detect faults and discontinuous change information ${ }^{[9]}$.

The angle detection technology is a calculation method which realized in Landmark,it calculates the angle changes between adjacent sampling points and interpret faults, according to the interpretation of a horizon information.

\section{APPLICATION EXAMPLES}

This study do interpret faults fine in bayachagan region using the several combining "body" faults'interpreting technique, at first, we need calculate various coherent body in PostStack/PAL of Landmark, e.g. Fig .1-4:

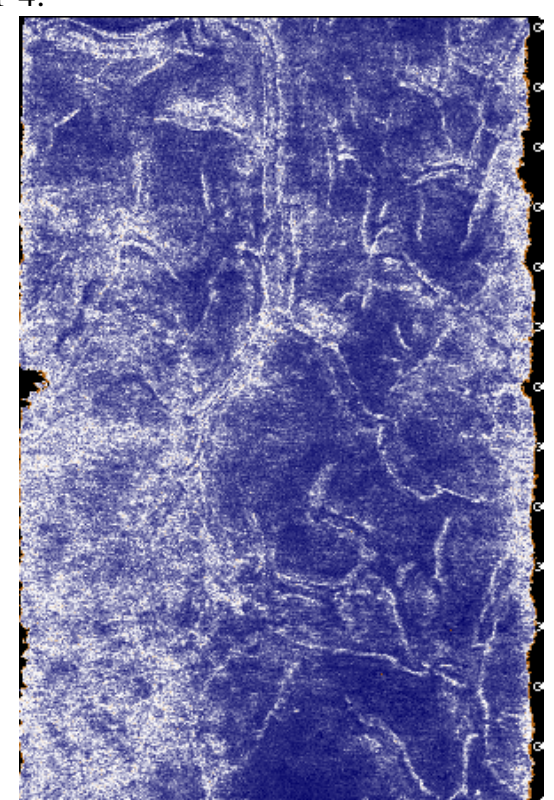

Fig .1 ESP_3D Along Horizon

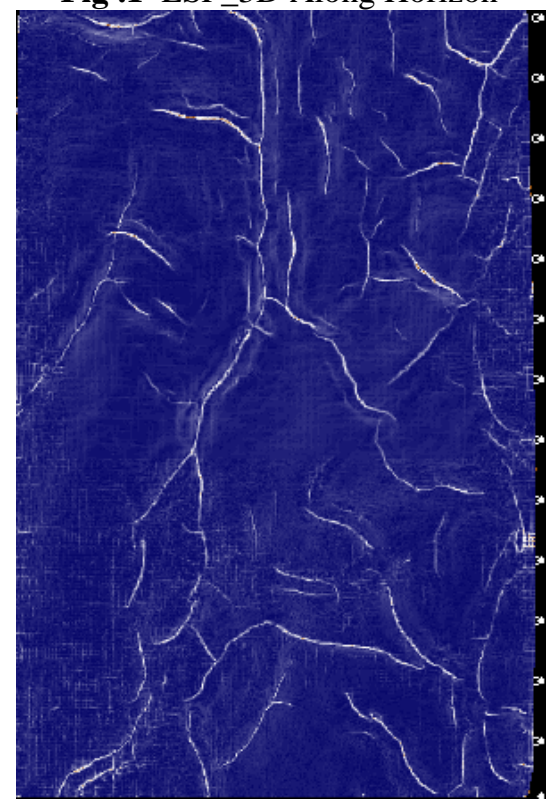

Fig. 3 Objective position to dip detection

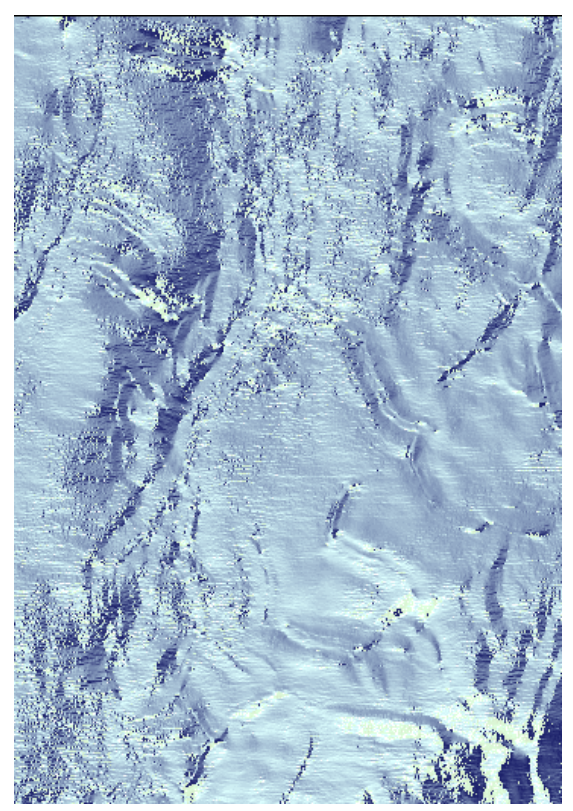

Fig. 2 Azimuth coherent body time slice

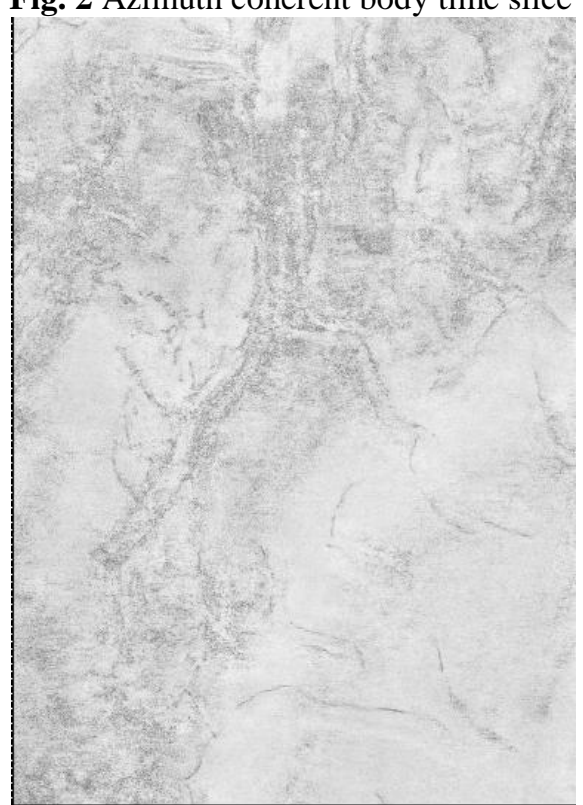

Fig. 4 The variance of coherence time slice

As can be seen from the graph, planar fault information reflected in each coherence is not exactly the same, so we had to need the interpreters to distinguish in section and check in space, so that get accurate faults. The best results of the above four chart is objective position to dip detection which identificats faults are more and clear, take \#1 fault as a example, e.g. Fig .5-8: 

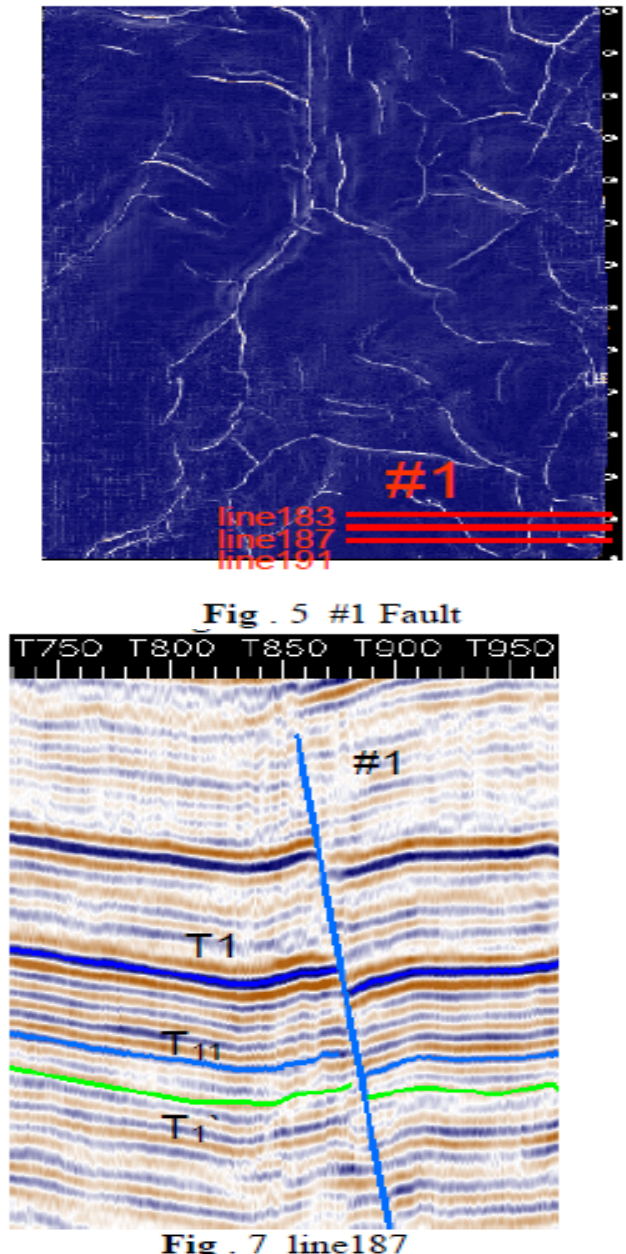
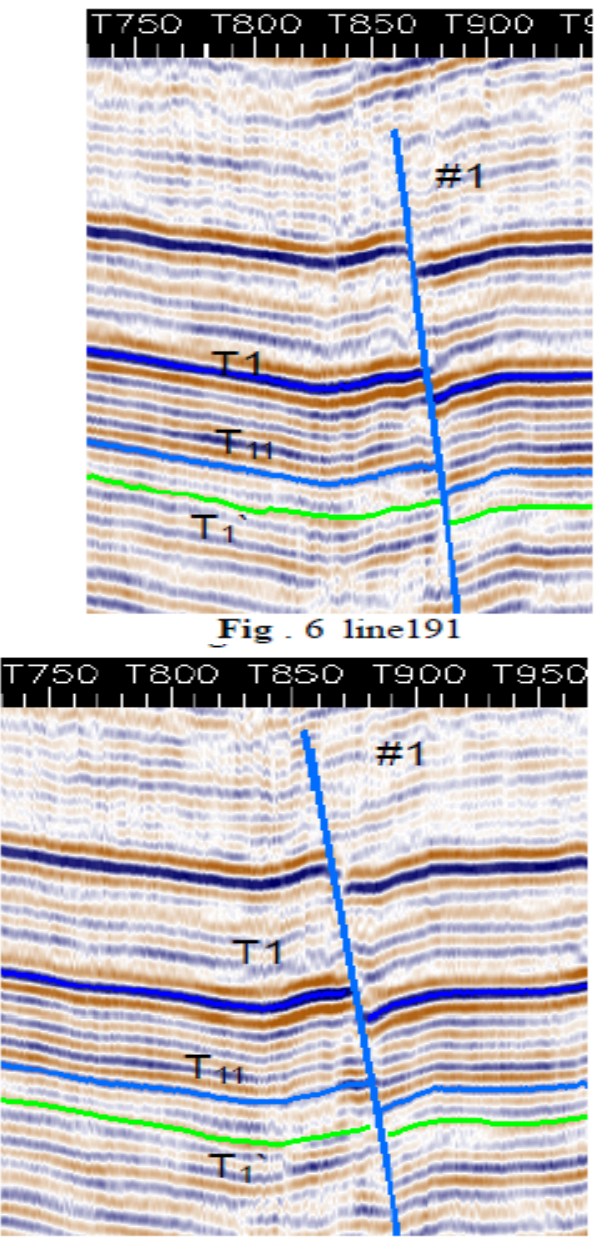

Fig - 8 line 183

Like this, we interpret each fault according to the principle of $4 * 4$, after interpreting the most faults, need compare with other coherent body slice, when Other coherent distinguished one fault while objective position to dip detection did not,we must do the above steps again and again, and tested whether the existence of this fault in section and space and closure of fracture surface, and in this way, this integrated of these coherent body together and picked up the fault information, minimized the number of missed fault.

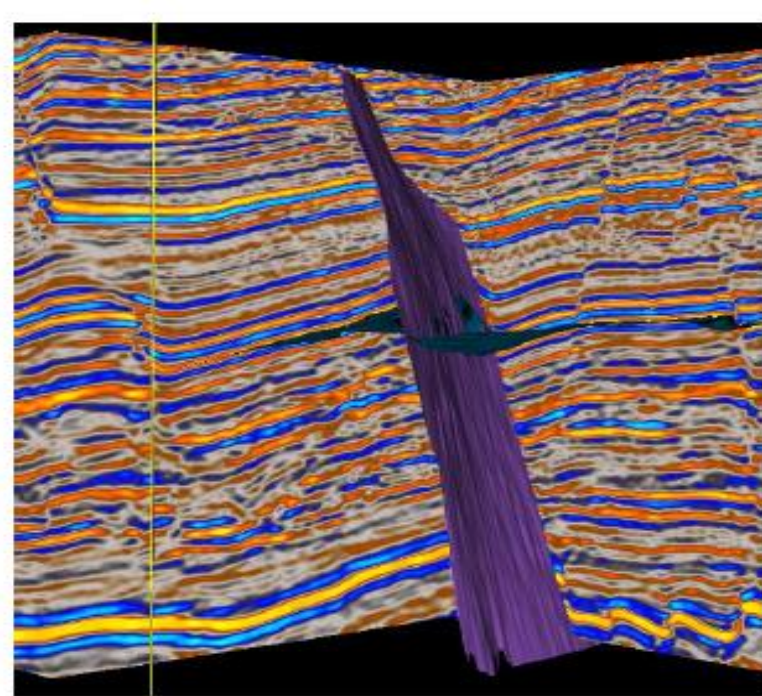

3d \#1 visualization

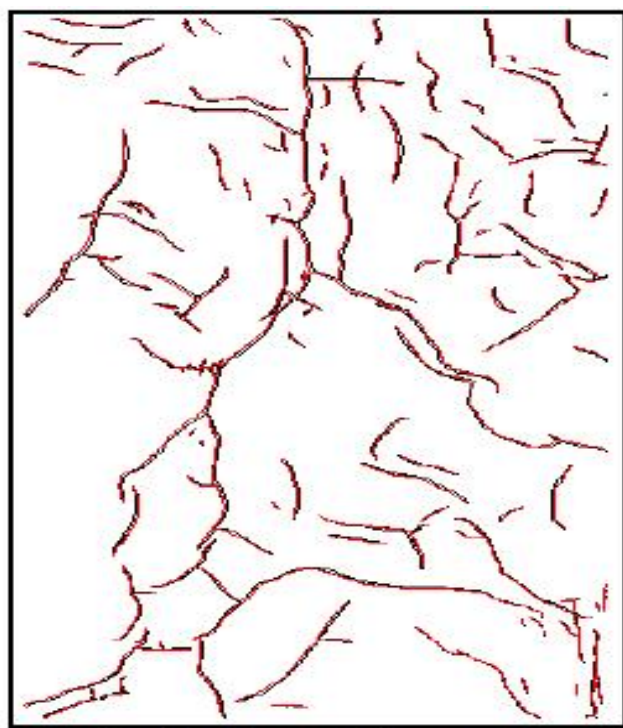

Faults interpretation result 
Esp_3d along horizon analysised and interpretated 105 faults, and the variance of coherence time slice analysised and interpretated 101 faults, and Azimuth coherent body time slice analysised and interpretated 85 faults, and objective position to dip detection analysised and interpretated 110 faults, but faults analysised by each coherent body are different, mutual authentication between every body, complement each other, after that, we can get 114 faults finally, through 3D visualization testing, faults interpretation is reasonable.

\section{CONCLUSION}

When only one coherent body information been used, we may explain faults more quickly, but slip some faults inevitably, while this four coherent body information been used together, not only improve the fault recognition ability and work efficiency but also reduces the interference of subjective factor, lay the foundation for the study on the characteristics of fault system.

\section{REFERENCES}

[1] $\mathrm{Hu}$ Wangshui,Lv Bingquan,Zhang Wenjun, Mao Zhiguo,Leng Jun,Guan Dayong. Evolution and basin forming dynamics of Songliao basin structure, Geological Science,2005,1(1):16 31.

[2] Zhao Wenzhi,Zou Caineng,Feng Zhiqiang,Hu Suyun,Zhang Yan,Li Ming,Wang Yuhua,Yang Tao, Yang Hui. Geological characteristics and evaluation technology of deep volcano rock gas in Songliao basin. Petroleum exploration and development,2008,4(2):129 142.

[3] Fu Xiaofei,Li Wenlong,Lv Yanfang,He Xiangyang,Liu Zhe. Fault lateral sealing and the control action of broken circle oil-water relationship. Geological review, 2011,3(57):386 397.

[4] Sun Yonghe,Han Yuping,Feng Zhipeng,Fu Xiaofei,Liu Zhe. Fracture system and the control action of oil and gas migration and gather in Hailar Basin. Geological review, 2011,1(57):89 100.

[5] Wang Yihan,Lu Shuangfang,Chen Fangwen,Han Jingjiang. Application of combination of well and seismic method in fine interpretation of faults, Science technology and Engineering, 2011,9(11):1922 1928.

[6] Xu Xikun,Wang Qinghua. Application of the eigen value of coherence technology in green east area, Journal of natural gas chemistry, 2008,06(30):265 269.

[7] Liao Lin, Yang Xiaomin. Application of coherence technique in Gudao area exploration[ A] 1 The technology of Shengli oilfield exploration set[ C] 1,BeiJing: Petroleum industry press, 2003:145 149.

[8] Wu Youxin,Fang Hanzhen. Application of coherent body and variance cube technique in the3D seismic data interpretation, Geology of Anhui, 2006,16(1):47 51.

[9] Qin Si, Zhao Xiansheng, Cheng $\mathrm{Hu}$, Wang Hongxia. Coherent and mechanism of crack detection algorithm of variance, Inner Mongolia petrochemical, 2007,12:81 85. 